\title{
SPECIAL MECHANISM OF CONDUCTION TYPE INVERSION IN PLASTICALLY DEFORMED $\boldsymbol{n}$-Si
}

\section{Teimuraz Pagava}

Department of Engineering Physics Georgian Technical University

77 M. Kostava ave., Tbilisi, Georgia, 0175

Levan Chkhartishvili

Department of Engineering Physics

Georgian Technical University

77 M. Kostava ave., Tbilisi, Georgia, 0175

levanchkhartishvili@gtu.ge

Manana Beridze

Department of Engineering Physics Georgian Technical University

77 M. Kostava ave., Tbilisi, Georgia, 0175

Magda Metskhvarishvili

Department of Engineering Physics

Georgian Technical University

77 M. Kostava ave., Tbilisi, Georgia, 0175

Iamze Kalandadze

Department of Engineering Physics

Georgian Technical University

77 M. Kostava ave., Tbilisi, Georgia, 0175

\section{Darejan Khocholava}

Department of Engineering Physics

Georgian Technical University

77 M. Kostava ave., Tbilisi, Georgia, 0175

\section{Nona Esiava}

Department of Engineering Physics

Georgian Technical University

77 M. Kostava ave., Tbilisi, Georgia, 0175

Maia Kevkhishvili

Department of Engineering Physics Georgian Technical University

77 M. Kostava ave., Tbilisi, Georgia, 0175

Marine Matcharashvili

Department of Engineering Physics

Georgian Technical University

77 M. Kostava ave., Tbilisi, Georgia, 0175

Abstract

The aim of research is studying the mechanism of $n-p$ inversion of the conduction type of deformed silicon crystals in the course of their thermal treatment. Initially, almost non-dislocation zone-melted phosphorus-doped n-Si single crystals with electron 
concentration of $2 \cdot 10^{14} \mathrm{~cm}^{-3}$ were studied. Uniaxial compression at temperature of $700{ }^{\circ} \mathrm{C}$ and pressure of $25 \mathrm{MPa}$ increased the dislocation density to $10^{8} \mathrm{~cm}^{-2}$. After long (within $30 \mathrm{~min}$ ) cooling of the deformed crystals to room temperature, an $n-p$ inversion of the conduction type occurred. The effect is explained by the formation of phosphorus-divacancy complexes $P V_{2}$ in the defective atmosphere of dislocations, which are acceptor centers with energy level of $E_{v}+0.34 \mathrm{eV}$. The found out n-p inversion mechanism differs from the standard one for plastically deformed n-type semiconductors with a diamond-like crystalline structure, which consists in the formation of acceptor centers along edge dislocations.

Keywords: conduction type inversion, plastic deformation, thermal treatment, silicon.

DOI: $10.21303 / 2461-4262.2019 .00938$

\section{Introduction}

Inversion of the type of conduction in semiconductors is an interesting physical effect, which has practical applications in solid-state electronics.

Usually, inversions to n-type are achieved by introducing acceptor impurities into the crystal and vice versa - by introducing donor atoms into the material with $p$-type conduction. An alternative direction to the chemical doping is the use of structural defects to influence the electrical properties of semiconductors. This approach, for its part, splits into the introducing (by irradiation, implantation, plasma treatment, annealing) of point and (by plastic deformation) extended defects of the crystal structure.

Briefly, the mechanism of the conduction type inversion induced by point defects can be described by the example of metal chalcogenides.

In the surface layer of $p$-type $\mathrm{Cd}_{x} \mathrm{Hg}_{1-x}$ Te samples $(x=0.22-0.25)$, when they were processed in hydrogen and/or argon plasma, an inversion of n-type conductivity was observed [1]. Despite the chemical activity of hydrogen, such inversion seems to be a purely radiation effect: excess mercury accumulated in micro-inclusions on the surface diffuses deep into the crystal and there suppresses defects of acceptor nature.

In the n-type $\mathrm{Cd}_{\mathrm{x}} \mathrm{Hg}_{1-\mathrm{x}}$ Te samples $(x=0.19-0.32)$, after implantation of ions of elements $\mathrm{I}$, III, and VIII groups and diffusion thermal annealing in atmosphere of saturated mercury vapor, an inversion to the p-type occurred [2]. The study of the recombination characteristics of such crystals has shown that, regardless of the electrical activity/neutrality of the implanted impurity their annealing leads to the formation of radiation defects of common nature. They should be vacancy complexes of the $V_{\mathrm{Hg}} V_{\mathrm{Te}}$ type in neutral state. The local levels in the band gap, through which carrier current recombines, are associated with same centers.

Inversion processes of the conduction type were also investigated [3] in the epitaxial films of the $p-\mathrm{Pb}_{0.8} \mathrm{Sn}_{0.2}$ Te solid solution under the action of laser radiation. It is also concluded that stable n-type inversion states arise due to the formation of bivacancies of metal and chalcogen.

Inversion of conduction from n- to p-type in zinc selenide samples doped with indium ZnSe:In was detected [4] during their annealing in atmosphere of saturated selenium vapor. The phenomenon was associated with the amphoteric nature of indium as dopant. In the process of annealing, a part of In atoms localized in the zinc sublattice goes out into the crystal lattice interstitials. Their association leads to the formation of micro-defects in the form of indium inclusions. These micro-defects serve as sources of effective diffusion of indium in selenium vacant sites, where they act as acceptors.

A generalized explanation of the inversion of the conduction type of metal chalcogenides is the following. These crystals are characterized, on the one hand, by high concentrations of electrically active vacancies of both metals and chalcogen, and, on the other hand, by metal-rich micro-inclusions. Stimulated by external influence (annealing, laser irradiation, ion implantation, etc.) processes of disintegration of micro-inclusions and diffusion of released atoms (especially under conditions of excessive pressure of metal vapor) can lead to change in the type of conduction from hole to electronic, since metal atoms when are filling vacancies in the chalcogen sublattice eliminate two free holes from each vacancy. At the same time, metal ions in interstitials are singly charged donors and for this reason the same processes occurred in neutral defective atmosphere can lead to the opposite direction of inversion. 
In most cases, the associated with point defects conduction type inversion occurs when the concentration of donors $n_{d}$ equals the concentration of acceptors $n_{a}: n_{d}=n_{a}$. And in the concentration region with center at $n_{d} \approx n_{a}$, Fermi level step of width $\sim k T / E_{g}$ is observed. Here $\mathrm{k}$ is the Boltzmann constant, $\mathrm{T}$ is the sample temperature, and $E_{g}$ is the band gap of the semiconductor. However, the condition $n_{d}=n_{a}$ is not always met. For example, in semi-insulating $p$-CdTe single crystals, the conduction type inversion was detected [5] at compensation degrees significantly lower than 1 . This and other features of the measured temperature-dependences of the electrical characteristics are explained by the special compensating mechanism in the semi-insulating material - in this case, by the presence of acceptor levels located above the middle of the band gap.

As for the inversion of the type of conduction associated with the introducing of extended defects into a semiconductor crystal, it has been studied in most detail for diamond-like structures. Unsaturated chemical bonds in the core of partial dislocations with an edge component in such a structure form acceptor centers with a system of electronic energy levels inside the band gap. Thus, high concentration of dislocations in diamond-like semiconductors always leads to the hole-type conduction.

Single-crystalline diamond, as is known, is an insulator, not a semiconductor. Since plastic deformation implies the appearance of both possible types of dislocations, significant plastic deformation of insulating diamonds results [6] in semiconductor diamonds, all of which without any exception have only the hole-type conduction.

As a criterion for the onset of the inversion of the conduction type associated with the introducing of dislocations into a semiconductor sample, the condition was proposed that differs from the one given above for the case when the inversion is caused by the introducing of point defects. Having in view diamond-like crystals, it is possible to formulate inversion criterion for $n$-type semiconductors in which dislocations with acceptor properties are introduced. Now the role of the concentration of acceptor centers is played by the ratio $n_{a D}=N_{D} / a$, where $N_{D}$ is the dislocations density and $a$ is the interatomic distance in the crystal. If the capacitance of the dislocation level closest to the valence band edge is sufficiently small, then with an increase in the density of dislocations such a value (again not necessarily equal to 1 ) of the ratio $n_{a D} / n_{d}$ is reached, which corresponds to a diffusive step at the Fermi level indicating the $n-p$ inversion of the conduction type.

For elementary semiconductors like germanium Ge and silicon $\mathrm{Si}$, which crystallize in diamond-like structure, the two-level phenomenological model was proposed [7]. In particular, in $n$-Si, a dislocation with an edge component must be charged and possess two acceptor levels, respectively, at distances $E_{1} \approx 0.42$ and $E_{2} \approx 0.67 \mathrm{eV}$ from the valence band edge. The capacity of the deeper level is always close to $1, C_{2} \approx 1$, and the capacity $C_{1}$ of another level may vary. If $C_{1}$ is sufficiently small $\left(C_{1} \sim 0.01\right)$, then with an increase in the density of dislocations at the Fermi level the step is formed with height of order of the difference $E_{2}-E_{1}$. This behavior is considered to be a sign of conduction type inversion.

The aim of this research is studying the mechanism of $n-p$ inversion of plastically deformed $n$-Si crystals during their thermal treatment; consideration of the possibility of interpreting this effect on the basis of the spectrum of complex point defects characteristic of the real structure of silicon.

\section{Experimental part}

Initially, obtained by zone-melting almost non-dislocation single crystals of $n$-Si doped with phosphorus of concentration of $2 \times 10^{14} \mathrm{~cm}^{-3}$ and with background oxygen content of $2 \times 10^{16} \mathrm{~cm}^{-3}$ have been studied.

Plastic deformation led to the dislocation multiplication with density up to $\sim 10^{8} \mathrm{~cm}^{-2}$. This value was determined by etching pits with the error of $\pm 5 \times 10^{6} \mathrm{~cm}^{-2}$. The deformation was performed by uniaxial compression acting in the main slip plane at temperature of $700{ }^{\circ} \mathrm{C}$ and pressure of $25 \mathrm{MPa}$.

After deformation, the samples were cooled slowly $(30 \mathrm{~min})$ together with the furnace or rapidly (30 s), for which they abruptly dropped into glycol. 
The carrier concentration $N$ was measured by the Hall method in the magnetic field of strength $10 \mathrm{kOe}$ in the temperature $T$ range from nitrogen boiling point to room temperature: $77-300 \mathrm{~K}$. Note that in the figure given in the next section, the experimental points for the low-temperature part of this range are not shown. The point is that, for low-conducting samples these measurements are impossible, and for highly-conducting ones the experimental values of the charge carriers' concentration are almost indistinguishable from each other. The Hall factor was assumed to be 1 .

The electronic energy levels of defects were determined from the slope of the dependences $\lg N-10^{3} / T$.

\section{Results}

The temperature-dependence of the electrons concentration (Fig. 1) for the initial sample in the entire measurement interval corresponds to the depletion of phosphorus dopant atoms by electrons: $N=$ const $\approx 2 \times 10^{14} \mathrm{~cm}^{-3}$ (Curve 1).

After plastic deformation with cooling for $30 \mathrm{~min}$, n-type samples with $\mathrm{N}_{\mathrm{D}} \sim 10^{8} \mathrm{~cm}^{-2}$ turned into p-type. The holes concentration in the valence band was determined by the ionization of centers with energy of $E_{v}+0.34 \mathrm{eV}$ (Curve 2).

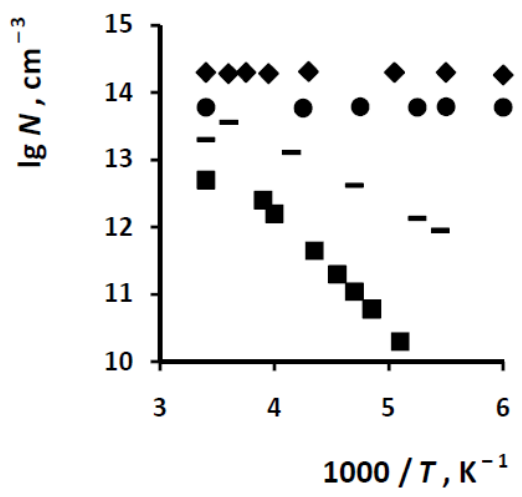

Fig. 1. Temperature-dependence of charge free carriers concentrations in plastically deformed silicon: 1 - before deformation $(\diamond)$; after deformation at $700{ }^{\circ} \mathrm{C}$ and 2 - cooling for $30 \mathrm{~min}(\mathbf{\square})$, 3 - annealing for $1 \mathrm{~min}$ at $700{ }^{\circ} \mathrm{C}$ and rapid cooling in glycol $(-)$, and $4-$ annealing at $700{ }^{\circ} \mathrm{C}$ for $\geq 35 \min (\bullet)$

All possible non-equilibrium defects in n-Si crystals, which can be formed by vacancies and impurity atoms during their plastic deformation and cooling, are stable up to $600{ }^{\circ} \mathrm{C}$. Therefore, by heating to $700{ }^{\circ} \mathrm{C}$ it is possible to ensure their complete dissociation. As for the equilibrium concentration of intrinsic point defects in silicon crystals at $700{ }^{\circ} \mathrm{C}$, it is about $10^{14} \mathrm{~cm}^{-3}$.

If both the equilibrium and released after 1 min annealing vacancies are fixed in nonequilibrium state by rapid cooling, the crystal again becomes of n-type and the electron concentration is determined by ionization of centers with energy $E_{c}-0.18 \mathrm{eV}$, which corresponds to A-centers, i. e. complexes of oxygen with vacancy OV (Curve 3).

After prolonged (for $\geq 35 \mathrm{~min}$ ) annealing at $700{ }^{\circ} \mathrm{C}$, the concentration of electrons in the conduction band is again determined by the shallow donors (Curve 4).

\section{Discussion}

Before discussing our results on the inversion of the type of conduction in n-Si, it is advisable to present the results of the work [8], in which the same effect was studied in highly pure material irradiated with $\gamma$-quanta. In the process of irradiating the samples, the electron concentration was first reduced to its intrinsic value, and then inversion of conduction into the p-type together with corresponding increase in the holes concentration occurred. The Fermi level as a result of $\gamma$-irradiation in highly pure silicon tends to the position at $E_{v}+0.39 \mathrm{eV}$ in the band gap. The following important conclusion from this result is that the inversion of the conduction type in $n$-Si is not necessarily associated with dislocation acceptors, but may be due to point defects. 
Similarly, the role of plastically deformed dislocations introduced into our samples does not seem to be decisive. This conclusion is supported by the possibility of n-type conduction recovery by thermal treatment, in which dislocations are not annealed. In the samples tested by us, the effective concentration of dislocation acceptors is insufficient for $n-p$ inversion. This value can be easily estimated from the difference in the electron concentrations in initial, i. e. not deformed, and deformed and continuously annealed samples (Curves 1 and 4): $1.4 \times 10^{14} \mathrm{~cm}^{-3}$.

In addition to the above, when interpreting the results obtained it is necessary to take into account that, during plastic deformation in silicon crystals along with each dislocation the ambient atmosphere of point defects are formed, in which the concentration of vacancies that actively interact with impurity atoms is particularly high [9].

When cooling plastically deformed samples for $30 \mathrm{~min}$ in the initial stages of the process (up to $300{ }^{\circ} \mathrm{C}$ ), it seems that only A-centers, i. e. complexes $\mathrm{OV}$, are formed. It is assumed that further on the basis of the A-centers there are formed deep complex defects also containing oxygen atoms and vacancies, for example, $O V_{n}$ and $O_{n} V(n=1,2,3)$ with ionization energies $\geq 0.44 \mathrm{eV}$, which are characterized by high thermal stability (with annealing temperature $\geq 350{ }^{\circ} \mathrm{C}$ ) [10].

In the temperature region around $250^{\circ} \mathrm{C}$, divacancies $\left(E_{c}-0.39 \mathrm{eV}\right)$ and defects with energy level $E_{c}-0.54 \mathrm{eV}$ are predominantly formed. The temperature ranges of annealing of these two defects almost coincide: $250-350{ }^{\circ} \mathrm{C}$ [11]. When the annealing temperature is $\leq 150{ }^{\circ} \mathrm{C}$, defects with low thermal stability can be formed in the crystal, for example, E-centers - complexes of doping phosphorus atoms with vacancies $P V$. They are deep acceptors and electrically neutral at this temperature. Presumably, in the same temperature range, in the neutral charge state there are monovacancies, which correspond to the energy level $E_{c}-0.28 \mathrm{eV}[12]$.

By blocking the phosphorus atoms, each $E$-center from the conduction band captures only one electron [13]. At an annealing temperature $\leq 150{ }^{\circ} \mathrm{C}$, specifically in the region of $100{ }^{\circ} \mathrm{C}$, negatively charged acceptor centers $P V_{2}^{-}$can be formed with the capture of an additional electron (by the reaction $2 E+V \rightarrow P V_{2}^{-}+P^{+}$), which corresponds to the energy level $E_{v}+0.34 \mathrm{eV}$ and are annealed in the temperature range of $400-500{ }^{\circ} \mathrm{C}$ [14]. It seems that, the ionization of just these centers determines the change in the holes concentration in the valence band, which leads to $n-p$ inversion of the conduction type during the thermal treatment of plastically deformed $n$-Si samples.

The $\mathrm{PV}_{2}$ complexes are formed at the end of the crystal cooling process when all possible deep acceptor centers have been already formed ( $E$-centers, divacancies, $V_{n} O$ - or $O{ }_{n} V$-type complexes, defect of unknown nature with the level of $E_{c}-0.54 \mathrm{eV}$, etc.).

At the time of the formation of the $P V_{2}$ complexes, all the electrons from the conduction band are already captured by the deep centers listed above. In order for these electrons to go to the level of $E_{v}+0.34 \mathrm{eV}$, they must first free themselves, i. e. go to the conduction band, what is possible at temperature of $\sim 350 \mathrm{~K}$. Correspondingly, the $P V_{2}$ complexes cannot capture electrons from the conduction band at relatively low temperatures $(\sim 300 \mathrm{~K})$.

The transition of electrons from the valence band to the level at $E_{v}+0.34 \mathrm{eV}$ begins in the region of $200 \mathrm{~K}$. It leads to $n-p$ inversion of the conduction type of the studied samples (Curve 2). And the holes concentration in inverted samples in the range $77-300 \mathrm{~K}$ is determined by the concentration of E-centers converted into $\mathrm{PV}_{2}$ complexes.

\section{Conclusion}

Thus, in n-type single crystalline plastically deformed at $700{ }^{\circ} \mathrm{C}$ and slowly cooled to room temperature, the $n-p$ inversion occurs. The observed effect is explained by the formation of phosphorous-divacancy complexes $P V_{2}$ with an acceptor energy level $E_{v}+0.34 \mathrm{eV}$ in defective atmosphere of dislocations multiplied by plastic deformation. This is a special mechanism for the inversion of the conduction type in plastically deformed silicon, which is different from the standard one associated with dislocation acceptors.

\section{References}

[1] Dvurechenskij, A. V., Remesnik, V. G., Ryazantsev, I. A., Talipov, N. Kh. (1993). Conduction type inversion in plasma-treated $\mathrm{Cd}_{x} \mathrm{Hg}_{1-x}$ Te layers. Semiconductors, 27 (1), 168-171. 
[2] Ibragimova, M. I., Baryshev, N. S., Zhikharev, V. A., Khaybullin, I. B. (1995). Recombination characteristics of ion-implanted (I, III and VIII groups) and thermally annealed $\mathrm{Cd}_{x} \mathrm{Hg}_{1-x}$ Te crystals. Semiconductors, 29 (10), 1755-1763.

[3] Grekov, Y. B., Shlyakhov, T. A., Semikolenova, N. A. (1997). Inversion of the conduction type of epitaxial films of PbSnTe solid solutions under the influence of laser irradiation at subthreshold power. Semiconductors, 31 (8), 844-846. doi: https:// doi.org/10.1134/1.1187239

[4] Vaksman, Y. F., Nitsuk, Y. A., Purtov, Y. N., Shapkin, P. V. (2003). Inversion of conductivity type in ZnSe single crystals obtained by the method of free growth. Semiconductors, 37 (2), 145-147. doi: https://doi.org/10.1134/1.1548654

[5] Kosyachenko, L. A., Maslyanchuk, O. L., Melnychuk, S. V., Sklyarchuk, V. M., Sklyarchuk, O. V., Aoki, T. (2010). Features of the mechanism of electrical conductivity of semiinsulating CdTe crystals. Semiconductors, 44 (6), 699-704. doi: https:// doi.org/10.1134/s1063782610060023

[6] Samsonenko, S. N., Samsonenko, N. D., Timchenko, V. I. (2010). Dislocation electrical conductivity of plastically deformed natural diamonds. Semiconductors, 44 (9), 1140-1144. doi: https://doi.org/10.1134/s1063782610090058

[7] Shikina, Yu. V., Shikin, V. B. (1994). Inversion of conduction type in plastically deformed n-semiconductors. Semiconductors, 28 (4), 675-680.

[8] Konozenko, I. D., Semenyuk, A. K., Khivrich, V. I. (1969). Radiation Defects Created by Co60 $\gamma$-Rays in p- and n-Type Si of High Purity. Physica Status Solidi (b), 35 (2), 1043-1052. doi: https://doi.org/10.1002/pssb.19690350257

[9] Milevskii, L. S., Tkacheva, T. M., Pagava, T. A. (1975). Trapping and anomalous scattering of majority carriers by interacting centers in plastically deformed n-type silicon. Journal of Experimental and Theoretical Physics, 42 (6), 1084-1088.

[10] Pagava, T. A. (2006). Effect of irradiation temperature on the efficiency of introduction of multivacancy defects into n-Si crystals. Semiconductors, 40 (8), 890-892. doi: https://doi.org/10.1134/s1063782606080057

[11] Vavilov, V. S., Kiselev, V. F., Mukashev, B. F. (1990). Defekty v kremnii i na ego poverhnosti. Moscow: Nauka, 216.

[12] Lukjanitsa, V. V. (2003). Energy levels of vacancies and interstitial atoms in the band gap of silicon. Semiconductors, 37 (4), 404-413. doi: https://doi.org/10.1134/1.1568459

[13] Luganov, P. F., Lukashevich, T. A. (1988). Efficiency of formation of phosphorus-containing complexes by electron- and $\gamma$-irradiating of silicon. Semiconductors, 22 (11), 2071-2073.

[14] Emtsev, V. V., Mashovets, T. V. (1981). Primesi i tochechnye defekty v poluprovodnikah. Moscow: Radio i svyaz', 248. 